\title{
Segurança e Exequiibilidade da Ecocardiografia com Estresse pela Dobutamina Associada à Atropina
}

\author{
W ilson Mathias J r, Léa Paula Beneti, Fabio Cesar dos Santos, Renata Duprat, Ailton Beraldo, \\ Manoel Adan Gil, José Lázaro Andrade, Eulógio Martinez
}

São Paulo, SP

\begin{abstract}
Objetivo - Estudar a segurança e exeqüibilidade da ecocardiografia com estresse pela dobutamina associada à atropina (EEDA) em pacientes com doença arterial coronária $(D A C)$ suspeita ou conhecida.

Métodos - Estudados 3000 testes consecutivos, pela EEDA, armazenados em formato de banco de dados.

Resultados - Observaram-se, como maiores complicações relacionadas ao teste, a ocorrência de 1 caso de infarto agudo do miocárdio, 4 de taquicardia ventricular sustentada e 5 de intoxicação atropínica. Um dos objetivos do teste foi atingido em $95 \%$ dos exames.

Conclusão - A EEDAé um método seguro e exeqüível para o diagnóstico de DAC.
\end{abstract}

Palavras-chaves: ecocardiografia, coronariopatia, dobutamina

\section{Safety and Feasibility of Dobutamine- Atropine Stress Echocardiography}

Purpose - To study the safety and feasibility of dobutamine-atropine stress echocardiography (DASE) in patients with known or suspected coronary artery disease.

Methods - There were 3,000 DASE that were studied in a prospective fashion. All symptons and side effects were stored in a data base format.

Results - Major test-related complications observed included one case of myocardial infarction, four cases of sustained ventricular tachycardia and five cases of atropine intoxication. There was no death or ventricular fibrillation as a direct or indirect consequence of the test. The infusion protocol allowed to us to examine patients using beta blockers, and led to 95\% feasibility.

Conclusion - DASE is a safe and feasible method for the study of patients with suspected or known coronary artery disease.

Key-words: echocardiography, coronary artery disease, dobutamine

Arq Bras Cardiol, volume 69 (n' 1$), 31-34,1997$

A dobutamina, sintetizada em $1975^{1}$, é uma catecolamina bem tolerada, possui meia-vida de 2 a 3 min e é uma das que mais aumentam o consumo de oxigênio pelo miocárdio, podendo, portanto, provocar isquemia. Estas características fizeram com que a mesma fosse possivelmente ideal, para provocar estresse cardíaco.

A ecocardiografia com estresse pela dobutamina foi utilizada pela primeira vez em 1986 para a avaliação da localização e extensão da doença arterial coronária (DAC), precocemente, após o infarto agudo do miocárdio (IAM), em 30

Escola Paulista de Medicina-UNIFESP e Hospital Unicor - São Paulo Correspondência: Wilson Mathias Jr - Rua Itapeva, 500 - Cj 5C - 01332-902 - São Paulo, SP

Recebido para publicação em 3/3/97

Aceito em 21/5/97 pacientes. Foi observado excelente perfil de tolerabilidade, com sensibilidade global de $85 \%$ e especificidade global de $88 \%$ para detecção de doença coronária distante da área de infarto $^{2}$. Desde então, vários outros trabalhos têm demonstrado sua alta acurácia diagnóstica e a importância de se adicionar atropina à dobutamina, a fim de aumentar o poder diagnóstico deste teste, especialmente nos pacientes em uso de drogas betabloqueadoras ${ }^{3,4}$.

Grandes séries são necessárias para que se estabeleça a segurança na prática médica de qualquer método terapêutico ou diagnóstico. Existem apenas três estudos na literatura médica, analisando a segurança da ecocardiografia pela dobutamina totalizando 4717 exames, com 10 complicações cardíacas significativas, porém sem nenhum óbito ${ }^{5-7}$. A ecocardiografia com estresse pela dobutamina não é isenta de efeitos colaterais significativos, que possam gerar risco 
de vida, porém, também não parece ser um método que gere número significativo de complicações. Portanto, pode-se observar que ainda não existem trabalhos suficientes para analisar de forma definitiva a segurança do teste.

Nosso objetivo foi avaliar a segurança e exeqüibilidade da ecocardiografia com estresse pela dobutamina associada à atropina (EEDA), assim como a utilidade da associação de atropina em 3000 testes consecutivos.

\section{Métodos}

No período de junho/91 a janeiro/97, 3000EEDA foram feitos em 2925 pacientes de forma consecutiva no Hospital Unicor e Hospital São Paulo da Escola Paulista de Medicina. Após a análise dos testes, os resultados foram registrados em banco de dados, utilizando planilha Excel da Microsoft com 114 campos de informação por paciente.

Foram estudados 1811 homens e 1189 mulheres, com idade média de 56,4 (3 a 89) anos, sendo que 838 submeteram-se ao exame, enquanto se encontravam internados e 2162 fizeram exames em âmbito ambulatorial. A história clínica demonstrava que 1589 eram hipertensos, 1375 tinham história de DAC em membros de $1^{\circ}$ grau da família <60 anos, 1047 hipercolesterolemia e 428 eram diabéticos.

Nos 3000 testes, os pacientes estudados possuíam uma ou mais das seguintes características clínicas pregressas: estavam em período após infarto do miocárdio recente (menos que duas semanas) (239); já haviam tido pelo menos um episódio de infarto do miocárdio antigo (maior que duas semanas) (700); tinham sido submetidos à revascularização do miocárdio (344); tinham sido submetidos à angioplastia transluminal coronária (477); apresentavam grau severo de disfunção ventricular esquerda (246) e houve 9 crianças estudadas a fim de se avaliar a presença de isquemia e viabilidade miocárdica por presença de fístulas coronárias, origem anômala de coronária e arterite de Takayasu. Como não foi solicitada a suspensão de medicamentos, 1042 pacientes estavam em uso de nitratos; $600 \mathrm{em}$ uso de bloqueadores dos canais de cálcio e 688 em uso de betabloqueadores.

Os critérios de inclusão foram: 1) pacientes com quadro clínico de insuficiência coronária e impossibilitados de realizar teste ergométrico, ou então, o realizaram de forma ineficaz por razões ortopédicas, neurológicas, vasculares ou idade avançada; 2) avaliação de viabilidade miocárdica; 3) pacientes restritos ao leito; 4) pacientes com quadro clínico sugestivo de insuficiência coronária e teste ergométrico negativo ou duvidoso; 5) pacientes assintomáticos com teste ergométrico positivo ou duvidoso; 6) presença, no eletrocardiograma (ECG), de bloqueios de ramo; 7) presença de infradesnivelamento de segmento ST em repouso, sem quadro clínico de insuficiência coronária significativa; 8) pacientes em uso de drogas podendo alterar o resultado do teste ergométrico; 9) estratificação de risco na fase subaguda do infarto do miocárdio não complicado; 10) acompanhamento após realização de angioplastia ou revascularização do miocárdio; e os de exclusão - 1) doença valvular grave; 2) quadro clínico instável; 3) pacientes em período após IAM complicado; 4) angina instável; 5) hipertensão arterial sistêmica não controlada; 6) arritmia ventricular grave ou de difícil controle; 7) gravidez; 8) alergia à dobutamina. Contra-indicações para o uso de atropina -1) glaucoma; 2) doença prostática.

Para que o teste fosse considerado eficaz, era necessário que se alcançasse um dos seguintes objetivos: 1) $85 \%$ da freqüência cardíaca máxima para a idade calculada pelo número 220 menos o valor numérico da idade; 2) observação de sinais de isquemia miocárdica; 3) término do protocolo de infusão. Caso ocorressem arritmias cardíacas graves ou efeitos colaterais significativos antes de se atingir um dos objetivos do teste, o mesmo era interrompido e considerado ineficaz.

Uma veia periférica era puncionada sempre com cateter venoso tipo Gelco $^{\mathrm{R}}$. Dois sistemas eram então instalados e conectados por torneira de dupla via; um com soro fisiológico a $0,9 \%$ para manutenção da permeabilidade venosa fora dos períodos de infusão de drogas, e outro, com solução formada por $230 \mathrm{~mL}$ de soro fisiológico a $0,9 \%$, no qual se adicionava $20 \mathrm{~mL}$ de solução contendo $250 \mathrm{mg}$ de dobutamina, resultando em uma concentração de $1 \mathrm{mg}$ de dobutamina por mililitro. A administração endovenosa de dobutamina era controlada por bomba de infusão, com doses crescentes, em estágios com 3min de duração, correspondentes a doses progressivas de $5 \mathrm{mcg} / \mathrm{kg} / \mathrm{min}$ até $40 \mathrm{mcg} / \mathrm{kg} / \mathrm{min}$. Caso até o $3^{\circ} \mathrm{min}$ do estágio de $40 \mathrm{mcg} / \mathrm{kg} /$ min, o paciente não apresentasse sinais de isquemia miocárdica evidentes ao ecocardiograma (ECO) e não tivesse atingido $85 \%$ da freqüência cardíaca máxima predita para a idade, atropina era administrada durante a infusão contínua de dobutamina, com doses de $0,25 \mathrm{mg}$ por minuto, até a dose máxima acumulativa de $1 \mathrm{mg}^{3}$. Havendo efeito colateral significativo ou isquemia miocárdica $5 \mathrm{mg}$ de metoprolol eram injetados via endovenosa (fig. 1). A cada estágio, determinavam-se a pressão arterial e a freqüência cardíaca. A monitorização eletrocardiográfica era feita em três derivações contínuas durante o teste. Os aparelhos utilizados na realização dos exames foram: Ultramark9 HDI, Ultramark9 Digital plus, Ultramark4 plus, Ultramark 8, Ultramark6eSIM7000.

Os sistemas de digitalização de imagens foram on line e off line da Novamicrosonics. As imagens ecocardiográficas gravadas em fita VHS, utilizando-se um VCR-

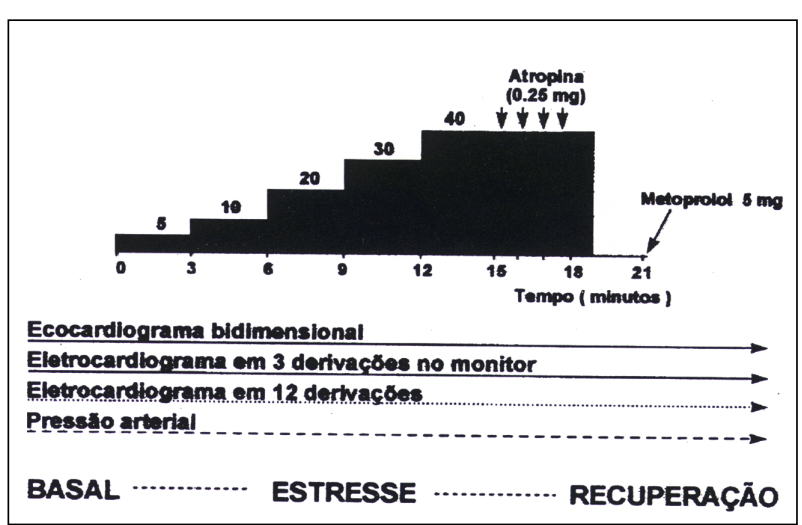

Fig. 1 - Protocolo de infusão de dobutamina associada à atropina 


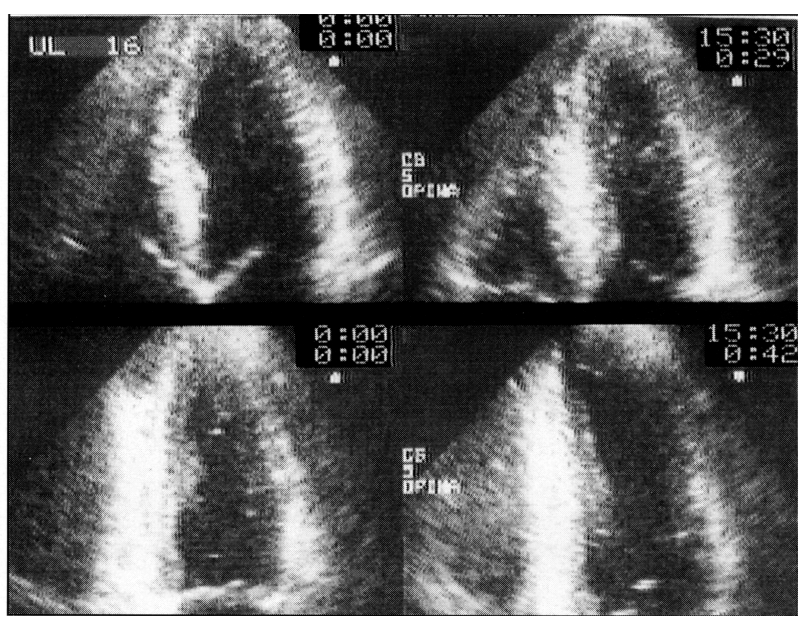

Fig. 2 - Imagem digital dos cortes apicais de duas (inferior) e quatro câmaras (superior) em repouso (direita) e no pico de infusão (direita) em um exame negativo para isquemia.

Panasonic e digitalizadas lado a lado (fig. 2). O ventrículo esquerdo foi avaliado em quatro planos ecocardiográficos padrões: paraesternal longitudinal, paraesternal transversal ao nível dos músculos papilares e apical quatro e duas câmaras, divididos em 16 segmentos como recomendado pela Sociedade Americana de Ecocardiografia ${ }^{8}$. A segurança do paciente na realização da EEDA foi avaliada em 3000 testes consecutivos.

Efeito colateral menor foi definido como aquele reversível em pequeno período de tempo e, potencialmente, incapaz de desencadear situação que levasse a risco de vida iminente. Hipotensão arterial foi definida como queda da pressão sistólica de pelo menos $20 \mathrm{mmHg}$ em relação à pressão sistólica de repouso e hipertensão arterial severa como pressão diastólica acima de $130 \mathrm{mmHg}$ e pressão sistólica acima de $240 \mathrm{mmHg}$. Efeito colateral maior foi definido como aquele que, potencialmente, pudesse gerar situação que levasse a iminente risco de vida. Efeito colateral com risco de vida foi definido como ao que levasse diretamente iminente risco de vida ao paciente.

Este estudo foi aprovado pelas comissões de ética médica da Escola Paulista de Medicina e do Hospital Unicor.

\section{Resultados}

Os objetivos do exame foram alcançados em $95 \%$ (2840/3000) dos testes, portanto, em 5\% dos casos o exame foi inconclusivo devido a efeito colateral limitante ou o exame foi interrompido precocemente a pedido do paciente.

Dos 3000 exames, 2027 (68\%) foram negativos e 973 (32\%) positivos para presença de isquemia miocárdica. Dos testes negativos, 1324 (65\%) não necessitaram de atropina para atingir um dos objetivos do teste e em 703 (35\%) a atropina foi necessária. Dos testes positivos, 701 (72\%) não precisaram da adição de atropina para se positivar, enquanto $272(28 \%) \mathrm{sim}$.

Os efeitos colaterais menores observados nos 3000 exames foram: precordialgia em $380(12,6 \%)$; hipotensão em 301 (10\%); náusea em $110(3,6 \%)$; hipertensão arterial severa em $69(2,3 \%)$; cefaléia em 56 (1,9\%); mal estar geral e tonturas em $21(0,7 \%)$; urgência miccional em $20(0,6 \%)$; dispnéia em $14(0,5 \%)$; tremores em $10(0,3 \%)$; vômitos em dois $(0,06 \%)$; tosse em um $(0,03 \%)$; astenia em um $(0,03 \%)$ e alergia leve em um $(0,03 \%)$. Hipotensão severa necessitando da infusão de solução fisiológica salina para recuperação dos níveis pressóricos basais em um exame negativo $(0,03 \%)$.

As arritmias observadas foram: extra-sistolia ventricular em 923 (31\%); extra-sistolia supraventricular em 296 $(9,9 \%)$; taquicardia ventricular não sustentada em 103 $(3,4 \%)$; bradicardia em $59(1,9 \%)$; taquicardia paroxística supraventricular em $23(0,8 \%)$; fibrilação atrial ou flutter atrial em $25(0,8 \%)$; ritmo juncional em nove $(0,3 \%)$; bloqueios atrioventriculares temporários em oito $(0,3 \%)$. Dois pacientes necessitaram amiodarona para reversão de fibrilação atrial e, um paciente, para um episódio de taquicardia paroxística supraventricular. Onze pacientes que apresentaram fibrilação atrial ou flutter atrial receberam metoprolol para a reversão da arritmia. Quatro pacientes com testes negativos para isquemia tiveram um episódio de taquicardia ventricular lenta sem comprometimento hemodinâmico, revertidos espontaneamente para ritmo sinusal. Os demais pacientes com outras arritmias cardíacas recobraram o ritmo sinusal com a interrupção da infusão de dobutamina ou após a administração de metoprolol.

Foram observados nove efeitos colaterais maiores, sendo cinco casos de intoxicação por atropina em indivíduos idosos que receberam dose total ( $1 \mathrm{mg}$ ) e apresentaram estado de torpor seguido por alucinações com duração de várias horas, necessitando de observação hospitalar por $24 \mathrm{~h}^{9,10}$ e quatro casos de taquicardia ventricular sustentada sem comprometimento hemodinâmico significativo, associados à presença de isquemia miocárdica. Em três houve a necessidade de metoprolol para reversão para ritmo sinusal e, em um, ela ocorreu de forma espontânea.

Também foi observado um caso de efeito colateral com risco de vida, 20min após o término da infusão de dobutamina, totalizando $0,17 \%$ de complicações cardíacas graves (efeito colateral maior ou com risco de vida). Tratava-se de paciente que apresentou IAM em parede anterior após exame em que se utilizou dose máxima de dobutamina associada a $0,5 \mathrm{mg}$ de atropina, atingindo a freqüência cardíaca de pico de $156 \mathrm{bpm}$. Não foram observadas alterações sugestivas de isquemia no ECG e no ECO. Vinte minutos após o término do exame, o paciente apresentou dor precordial súbita, acompanhada de supra desnivelamento do segmento ST ao ECG e discinesia de parede ântero-septo-apical ao ECO, necessitando de revascularização miocárdica de urgência. Permaneceu sem outras complicações até o último seguimento três anos após.

\section{Discussão}

A ecocardiografia com estresse em suas várias formas é um método relativamente novo, com pouco mais de 10 anos desde os primeiros relatos. Pode-se entender que dados relativos à segurança do mesmo não sejam tão extensos quanto aos já conhecidos em ergometria e em medicina nu- 
clear. A ecocardiografia com esforço, provavelmente, reflete os dados relativos à segurança do teste ergométrico, pois esta segue protocolos semelhantes aos padronizados no passado para essa técnica. É estimado que a cada 10.000 testes ergométricos haja três eventos graves $(0,03 \%)$ e um óbito como conseqüência ${ }^{11}$.

Não houve óbito secundário à EEDA relatado até o momento na literatura. Em estudo com 1118 indivíduos ${ }^{3}$ não se observaram complicações graves $(0 \%)$ e, em outro, foram avaliados 650 pacientes, tendo sido observado um caso de fibrilação ventricular $(0,15 \%)^{4}$. No estudo EDIC, (Echo Dobutamine International Cooperative Study) ${ }^{5}$ avaliando 2949 testes, observaram-se nove complicações cardíacas graves $(0,3 \%)$, sendo dois infartos, duas fibrilações ventriculares, duas hipotensões prolongadas, uma isquemia prolongada e dois casos de taquicardia ventricular. Não houve óbito.

No presente estudo, os efeitos colaterais menores, em sua grande maioria, desapareceram com a simples interrupção da infusão da droga. Houve cinco casos de indivíduos idosos que apresentaram quadro clínico de intoxicação atropínica, necessitando internação hospitalar. Todos os sintomas desapareceram num intervalo máximo de $24 \mathrm{~h}$. Como estes casos ocorreram em pessoas mais velhas que 58 anos de idade, a partir de 1994, não mais foram utilizadas doses maiores de $0,5 \mathrm{mg}$ em indivíduos acima dessa faixa etária. Também observamos quatro casos de taquicardia ventricular sustentada, um caso de IAM, resultando em uma incidência de eventos cardíacos graves $0,17 \%$. O caso de infarto pode ser explicado pelo fato de a dobutamina poder causar espasmo coronário e aumento da atividade plaquetária $^{12,13}$.

Também cabe enfatizar que não houve caso de fibrilação ventricular ou óbito, como conseqüência direta ou indireta do exame, no presente estudo.

A experiência de cada centro investigativo também deve representar papel importante no perfil de segurança e tolerabilidade dos testes de estresse, pois a mesma é diretamente relacionada à capacidade de o médico operador reconhecer imediatamente os sinais ecocardiográficos de isquemia miocárdica. A utilização de protocolos mais agressivos na infusão de dobutamina e sua associação com a atropina, a fim de se alcançar maior acurácia diagnóstica do exame, pode ter colaborado para o aparecimento de algumas complicações relatadas na literatura e também neste estudo.

No presente estudo, os efeitos colaterais menores em sua grande maioria desapareceram com a simples interrupção da infusão da droga. Houve cinco casos de indivíduos idosos que apresentaram quadro clínico de intoxicação atropínica necessitando internação hospitalar. Todos os sintomas desapareceram num intervalo máximo de 24 horas. Como estes casos ocorreram em pessoas mais velhas que 58 anos de idade, a partir de 1994 não mais foram utilizadas doses maiores de 0,5 mg em indivíduos acima desta faixa etária. Também observamos quatro casos de taquicardia ventricular sustentada, um caso de infarto agudo do miocárdio, resultando em uma incidência de eventos cardíacos graves $0,17 \%$. O caso de infarto pode ser explicado pelo fato de a dobutamina poder causar espasmo coronário e aumento da atividade plaquetária ${ }^{12,13}$.

Também cabe enfatizar que não houve caso de fibrilação ventricular ou óbito, como consequência direta ou indireta do exame no presente estudo.

A experiência de cada centro investigativo também deve representar papel importante no perfil de segurança e tolerabilidade dos testes de estresse, pois a mesma é diretamente relacionada à capacidade de o médico operador reconhecer imediatamente os sinais ecocardiográficos de isquemia miocárdica. A utilização de protocolos mais agressivos na infusão de dobutamina e sua associação com a atropina, a fim de se alcançar maior acurácia diagnóstica do exame pode ter colaborado para o aparecimento de algumas complicações relatadas na literatura e também neste estudo. A população estudada em cada trabalho também difere. No estudo EDIC ${ }^{5}, 43 \%$ dos pacientes estavam no período após infarto do miocárdio recente, ao passo que em nosso estudo, menos de $8 \%$ dos pacientes foram estudados nesse período.

Concluindo, a EEDA mostrou ser um método diagnóstico seguro e exeqüível, quando empregado ao nível ambulatorial e hospitalar em grande variedade de pacientes, com ou sem DAC. A adição de atropina foi necessária para que fosse atingido um dos objetivos, em $32 \%$ dos testes.

\section{Referências}

1. Turtle RR, Mills J - Dobutamine development of a new cathecholamine to increase cardiac contractility. Circ Res 1975; 36: 185-96.

2. Berthe C, Pierard SA, Hiernaux Met al - Predicting the extent and location of coronary artery disease in acute myocardial infarction by echocardiography during dobutamine infusion. Am J Cardiol 1986; 58: 1167-72.

3. MacNeill A, Fioretti PM, El Said EM et al - Enhanced sensitivity for detection of coronary artery disease by addition of atropine to dobutamine echocardiography. Am J Cardiol 1992; 70: 41-6.

4. Segar DS, Brown SE, Sawada SG et al - Dobutamine stress echocardiography: Correlation with coronary lesion severity as determined by quantitative angiography. J Am Coll Cardiol 1992; 19: 1197-202.

5. Mertes H, Sawada S, Ryan T et al - Symptons, adverse effects and complications associated with dobutamine stress echocardiography; experience in 1118 patients. Circulation 1993; 88: 15-19.

6. Poldermans D, Fiorette PM, BoersmaEet al. - Safety of dobutamine-atropine stress echocardiography in patients with suspected or proven coronary artery disease: experience in 650 consecutive examinations. Am J Cardiol 1994; 73: 456-9.

7. Picano E, Mathias W, Pingitore A et al - Safety and tolerability of dobutamine atropine stress echocardiography: a prospective, multicentre study. Lancet 1994; 344: 1190-2.

8. Shiller N, Shah P, Crawford Met al - Recommendations for quantitation of the left ventricle by two dimensional echocardiography. J Am Soc Echo 1989; 2: 358-67.

9. Ziskind AA - Transdermal scopolamine-induced psychosis. Postgrad Med 1988; 84: 73-6.

10. Wilkinson JA - Side effects of transdermal scopolamine. J Emerg Med 1987; 5: 389-92.

11. Stuart RJ, Ellestad MH - Natural survey of exercise stress testing facilities. Chest 1980; 77: 94.

12. Kawano H, Fuji H - Induction of coronary spasm during dobutamine stress echocardiography. Circulation 1995; 92(suppl I): nº 424.

13. ArenaF, Paglioroni T, Michael TW, Galloway T, Bommer WJ, Lewis WR - Platelet

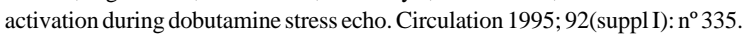

